\title{
(-)-Epigallocatechin-3-gallate induces interferon- $\lambda 2$ expression to anti-influenza A virus in human bronchial epithelial cells (BEAS-2B) through p38 MAPK signaling pathway
}

\author{
Jie $\mathrm{Zhu}^{1 "}$, $\mathrm{Li} \mathrm{Ou}^{2 \#}$, Yongjun $\mathrm{Zhou}^{3}$, Zixiao Yang ${ }^{4}$, Mingjiang $\mathrm{Bie}^{5,6}$ \\ ${ }^{1}$ Department of Microbiology, School of Basic Medical Science, Guizhou Medical University, Key Laboratory of Medical Microbiology and \\ Parasitology, Guizhou Province Department of Education, Guiyang 550025, China; ${ }^{2}$ Department of Pulmonary and Critical Care Medicine, \\ Affiliated Chengdu 363 Hospital of Southwest Medical University, Chengdu 610041, China; ${ }^{3}$ Department of Microbiology, West China School of \\ Basic Medical Sciences and Forensic Medicine, Sichuan University, Chengdu 610041, China; ${ }^{4}$ Department of Clinical Medicine, North Sichuan \\ Medical College, Nanchong, China; ${ }^{5}$ West China School of Public Health and West China Fourth Hospital, Sichuan University, Chengdu 610041, \\ China; ${ }^{6}$ Editorial Board of Journal of Sichuan University (Medical Science Edition), Chengdu 610041, China \\ Contributions: (I) Conception and design: J Zhu, L Ou, M Bie; (II) Administrative support: None; (III) Provision of study materials or patients: \\ None; (IV) Collection and assembly of data: Y Zhou, Z Yang; (V) Data analysis and interpretation: J Zhu, L Ou, M Bie; (VI) Manuscript writing: All \\ authors; (VII) Final approval of manuscript: All authors. \\ \#These authors contributed equally to this work. \\ Correspondence to: Mingjiang Bie. West China School of Public Health and West China Fourth Hospital, Sichuan University, Chengdu 610041, \\ China; Editorial Board of Journal of Sichuan University (Medical Science Edition), No. 17, 3rd Section, Chengdu 610041, China. \\ Email: 13941057@qq.com.
}

Background: (-)-Epigallocatechin-3-gallate (EGCG), a major component of green tea, has been found to inhibit the influenza virus. However, the mechanism of EGCG anti-influenza virus effect needs to be further explored.

Methods: BEAS-2B cells were treated with different concentrations of EGCG or were treated with EGCG for different times. CCK8 assay was used to detect the cell viability, and quantitative real time polymerase chain reaction (qRT-PCR) and enzyme-linked immunosorbent assay were employed to measure the interferon (IFN)- $\lambda 2$ mRNA and protein expression levels. The phospho-p38 mitogen-activated protein kinase (P-p38 MAPK), phospho-extracellular signal-regulated kinase (P-ERK), and phospho-c-Jun N-terminal kinase (P-JNK) expression were tested by western blot. Then, p38 MAPK, ERK, and JNK inhibitor were used to study the effect of p38 MAPK, ERK, and JNK signaling pathways on IFN- $\lambda 2$ expression. The BEAS-2B cells were treated with EGCG, EGCG and IFN $\lambda 2$ neutralizing antibody or control antibody for $12 \mathrm{~h}$, and were infected with influenza A virus (IAV) (H1N1) for $1 \mathrm{~h}$. After $12 \mathrm{~h}$, nucleoprotein (NP) mRNA and protein expression levels of H1N1 were assessed by qRT-PCR and western blot.

Results: The IFN- $\lambda 2$ mRNA and protein expression levels in BEAS-2B cells were up-regulated after EGCG (treatment in time- and dose-dependent manners the concentration range from 0 to $50 \mu \mathrm{g} / \mathrm{mL} \mathrm{had}$ no cytotoxicity). Meanwhile, the P-p38 MAPK, P-ERK, and P-JNK expression levels were up-regulated. IFN- $\lambda 2$ mRNA and protein expression was inhibited after p38 MAPK inhibitor pre-treatment, but not by ERK and JNK inhibitors. Furthermore, the expression of H1N1 NP gene and protein decreased after EGCG pre-treatment, while IFN- $\lambda 2$ neutralizing antibody attenuated the effect of EGCG inhibiting the expression of H1N1 NP gene and protein.

Conclusions: EGCG inhibited IAV H1N1 by inducing the expression of IFN- $\lambda 2$ in BEAS-2B cells through the p38 MAPK signaling pathway.

Keywords: (-)-Epigallocatechin-3-gallate (EGCG); interferon- $\lambda 2$ (IFN- $\lambda 2$ ); p38 mitogen-activated protein kinase (p38 MAPK); influenza A virus (IAV) 
Submitted Feb 20, 2020. Accepted for publication Mar 18, 2020.

doi: $10.21037 /$ jtd.2020.03.20

View this article at: http://dx.doi.org/10.21037/jtd.2020.03.20

\section{Introduction}

The influenza virus is a single-stranded negative-sense RNA virus belonging to the Orthomyxoviridae family, and can be divided into 4 types: of $\mathrm{A}, \mathrm{B}, \mathrm{C}$, and $\mathrm{D}(1,2)$. Among these types, the influenza A virus (IAV) is the dominant pathogen. IAV is prone to antigenic variation, so it is seasonally prevalent every year. According to the latest report, influenza causes 290,000 to 650,000 deaths every year, and is a serious threat to human health (3). A universal influenza vaccine has not yet been developed although influenza typeand subtype-specific vaccine can be developed. Given this, considerable time and money have been expended to predict the prevalent strains and formulate effective vaccines (4). Among these measures, adamantanes and neuraminidase (NA) inhibitors are 2 classes of antiviral drugs that have been approved for treatment of influenza virus infections. However, influenza virus resistance to these drugs is a significant concern (5-7). There is therefore an urgent need for an effective strategy that can control the influenza virus.

Respiratory epithelium cells serve as the first line of defense against the influenza virus and culminate in the production of interferon (IFN), a key molecule in the antiviral innate immune response $(8,9)$. However, the virulence factors of the influenza virus can antagonize the IFN immune response and promote influenza virus infection (10). Therefore, inducing IFN expression may be an effective strategy against the influenza virus. (-)-Epigallocatechin-3-gallate (EGCG) is the most abundant and bioactive catechin in green tea (11). Previous studies have revealed that EGCG exhibits affects against viral infection, including against the human immunodeficiency virus (HIV) (12), the herpes simplex virus (HSV) (13), the hepatitis B virus (HBV) (14), the hepatitis C virus (HCV) (15), and the Zika virus (16). It has been shown that EGCG enhances virus dsRNA or poly I:C-induced IFN- $\lambda 1$ production and inhibits $\mathrm{HCV}$ replication $(17,18)$. Although many studies have reported that EGCG has anti-influenzavirus effects (19), whether EGCG can induce IFN- $\lambda$ expression in airway epithelial cells to suppress the influenza virus and related signaling pathways has not been elucidated at this stage.

In this study, we used the tracheal epithelial cells (BEAS2B) as model to investigate EGCG's anti-IAV effects and mechanisms. The results confirmed that EGCG can induce IFN- $\lambda 2$ expression through p38 MAPK signaling pathway, but not through the ERK and JNK signaling pathways. It was also found that IFN- $\lambda 2$ neutralizing antibody attenuated the role of EGCG inhibiting the expression of H1N1 nucleoprotein (NP) gene and protein. These findings provide some insight into the mechanisms behind EGCG's anti-IAV (H1N1) activity.

\section{Methods}

\section{Cell and virus culture}

Human bronchial epithelial cells (BEAS-2B) and MadinDarby canine kidney (MDCK) cells were purchased from the Kunming Cell Bank of the Chinese Academy of Sciences (Kunming, China). All the cells were cultured in Dulbecco's Modified Eagle's Medium (DMEM; Gibco, Grand Island, NY, USA) with 10\% fetal bovine serum (FBS) (Gibco, Rockville, MD, USA) and 1\% penicillin and streptomycin solution (HyClone, Logan, UT, USA) at $37{ }^{\circ} \mathrm{C}$ in an atmosphere of $5 \% \mathrm{CO}_{2}$.

IAV samples (A/PR/8/34 H1N1, PR8) were purchased from the National Influenza Center, National Institute for Viral Disease Control and Prevention Chinese Center for Disease Control and Prevention (Beijing, China), and were amplified in the allantoic cavity of embryonated eggs for $48 \mathrm{~h}$ at $37{ }^{\circ} \mathrm{C}$, and were stored after at $-80^{\circ} \mathrm{C}$. Viral titers were determined by calculating the $50 \%$ tissue culture infectious dose $\left(\mathrm{TCID}_{50}\right)$ per milliliter using the ReedMuench method in MDCK cells.

\section{Treat BEAS-2B cells}

BEAS-2B cells were added into $96-$ well plates $\left(1 \times 10^{4}\right.$ cells/well) or 6 -well plates $\left(5 \times 10^{5}\right.$ cells/well). After this, the BEAS$2 \mathrm{~B}$ cells in 96-well plates were treated with different concentrations (i.e., 0, 6.25, 12.5, 25, 50, and $100 \mu \mathrm{g} / \mathrm{mL}$ ) of EGCG (Solarbio, Beijing, China) for $48 \mathrm{~h}$. The BEAS$2 \mathrm{~B}$ cells in the 6 -well plates were treated with different concentrations (i.e., 0, 6.25, 12.5, 25 and $50 \mu \mathrm{g} / \mathrm{mL}$ ) of EGCG for $12 \mathrm{~h}$; were treated with EGCG $(50 \mu \mathrm{g} / \mathrm{mL})$ for 0 , 2, 4, 12, 24, and $48 \mathrm{~h}$; or were incubated with $20 \mu \mathrm{M}$ of p38 MAPK inhibitor (SB203580, Beyotime, ShangHai, China), 
$20 \mu \mathrm{M}$ of ERK inhibitor (U1026, Beyotime, Shanghai, China), or $10 \mu \mathrm{M}$ of JNK inhibitor (SP600125, Beyotime, Shanghai, China) dissolved in dimethyl sulfoxide (DMSO) for $1 \mathrm{~h}$, with the supernatant being removed and mixed with EGCG $(50 \mu \mathrm{g} / \mathrm{mL})$ for $4 \mathrm{~h}$. The BEAS-2B cells in 6-well plates were treated with EGCG $(50 \mu \mathrm{g} / \mathrm{mL})$, EGCG $(50 \mu \mathrm{g} / \mathrm{mL})$ and IFN $\lambda 2$ neutralizing antibody $(0.5 \mu \mathrm{g} / \mathrm{mL}$, MAB1587, R\&D, Minneapolis MN, USA) or control antibody $(0.5 \mu \mathrm{g} / \mathrm{mL}, \mathrm{MAB} 002, \mathrm{R} \& \mathrm{D}$, Minneapolis $\mathrm{MN}$, USA) for $12 \mathrm{~h}$. Next, the supernatant was removed, and cells were infected with IAV (H1N1) for $1 \mathrm{~h}$ at $37^{\circ} \mathrm{C}$. After the supernatant was removed, the cells were washed twice and recovered with DMEM to culture for $12 \mathrm{~h}$.

\section{Cell viability assessment}

The BEAS-2B cells in 96-well plates were treated with different concentrations EGCG for $48 \mathrm{~h}$. After that, $20 \mu \mathrm{L} /$ well Counting Kit-8 (CCK8) (Dojindo, Kumamoto, Japan) was added into each well and incubated for $2 \mathrm{~h}$ at $37^{\circ} \mathrm{C}$. Then, the absorbance was read at $450 \mathrm{~nm}$ on a microplate reader (Bio-Tec Instruments, Burlington, VT, USA). The cell viability rate was calculated using the following formula: cell viability rate $=\left[\left(\mathrm{OD}_{\mathrm{EGCG}(0-100 \mu / \mathrm{mL})}-\mathrm{OD}_{\text {zeroing }}\right) /\left(\mathrm{OD}_{\mathrm{EGCG}(0 \mathrm{\mu g} / \mathrm{mL})}\right.\right.$ $\left.-\mathrm{OD}_{\text {zeroing }}\right] \times 100 \%$.

\section{$R N A$ isolation and quantitative real time polymerase chain reaction ( $R$ RT-PCR)}

Total RNAs were isolated by using Total RNA extraction kit (Tian Gen, Beijing, China). The RNA purity and quantity were measured on NanoDrop 2000 spectrophotometer (Thermo Fisher Scientific, Waltham, MA, USA). Next, the PrimeScript ${ }^{\mathrm{TM}}$ RT reagent Kit with gDNA Eraser (Takara, Dalian, China) and total RNA template was used to synthesize the cDNA. The quantitative real-time polymerase chain reaction (qRT-PCR) was performed on CFX96 Real-Time PCR System (Bio-Rad, Hercules, CA, USA) by using the cDNA, SYBR Premix Ex Taq (Takara, Dalian, China), and the following pre-designed primers (GENEWIZ, Suzhou, China): IFN- $\lambda 2$ forward: 5'-GGGCCTGTATCCAGCCTCAG-3' and reverse: 5'-GAGGAGGCGGAAGAGGTTGA-3'. NP forward: 5'-GACGATGCAACGGCTGGTCTG-3' and reverse: 5'-AGCATTGTTCCAACTCCTTT-3'. GAPDH forward: 5'-AGAAGGCTGGGGCTCATTTG-3' and reverse: 5'-AGGGGCCATCCACAGTCTTC-3'.

GAPDH was used as the reference gene for normalizing and determining the fold change in the expression of IFN- $\lambda 2$ and NP mRNA, which was calculated using the 2(-Delta Delta Ct) method.

\section{Enzyme-linked immunosorbent assay}

After stimulation, the supernatants were gently collected to determine the protein concentrations of IFN- $\lambda 2$ using commercially available ELISA kits (Elabscience, Wuhan, China) according to the manufacturer's instructions.

\section{Western blot analysis}

Cells were washed with $37^{\circ} \mathrm{C}$ PBS, mixed with RIPA Lysis Buffer for $10 \mathrm{~min}$ at $4{ }^{\circ} \mathrm{C}$, and centrifuged to yield wholecell lysates. The protein sample $(20 \mu \mathrm{g})$ was separated by SDS polyacrylamide gels with electrophoresis (BioRad, USA), and the gel was transferred to polyvinylidene fluoride (PVDF) membranes (Millipore, Billerica, MA, USA). The membranes were blocked with blocking buffer for $2 \mathrm{~h}$ and then incubated overnight at $4{ }^{\circ} \mathrm{C}$ with the corresponding primary antibodies, including phosphoextracellular signal-regulated kinase (P-ERK) $(1: 1,000$ dilution, \#4370, CST, USA), ERK (1:1,000 dilution, \#4695, CST, USA), phospho-c-Jun N-terminal kinase (P-JNK) (1:1,000 dilution, ab124956, Abcam, Cambridge, UK), JNK (1:1,000 dilution, ab179461, Abcam, Cambridge, $\mathrm{UK})$, phospho-p38 mitogen-activated protein kinase (Pp38 MAPK) (1:1,000 dilution, \#9211, CST, USA), p38 MAPK (1:1,000 dilution, \#9212, CST, USA), NP (1:1,000 dilution, bs-4976R, Bioss, Beijing, China), and GAPDH (1:700, M00227-5, Boster, Wuhan, China). The membrane was washed with TBS-T and incubated with the HRP-goat anti-Rabbit IgG (1:5000 dilution, BA1054, Boster, Wuhan, China) as the secondary antibody and then developed in electrochemiluminescent western detection reagents (Millipore, Billerica, MA, USA).

\section{Statistical analysis}

Data are represented as means \pm standard deviations (SD) of results from the 3 independent experiments with similar patterns. Significant differences between the indicated groups were determined by Student's $t$-test and one-way analysis of variance (ANOVA) using GraphPad Prism 5.0 software (GraphPad, San Diego, CA, USA). For all experiments, $\mathrm{P}<0.05$ was considered a statistically significant value. 


\section{Results}

Cytotoxicity of EGCG on BEAS-2B cells

To confirm the cytotoxicity of EGCG on BEAS-2B cells, the BEAS-2B cells were treated with various concentrations (i.e., $12.5,25,50$, and $100 \mu \mathrm{g} / \mathrm{mL}$ ) of EGCG for $48 \mathrm{~h}$, and then CCK-8 detection kit was used to detect the viability of cells. The result suggests treatment with EGCG at the concentration range of 0 to $50 \mu \mathrm{g} / \mathrm{mL}$ for $48 \mathrm{~h}$ elicits no cytotoxicity on BEAS-2B cells (Figure 1). Thus, this concentration range was applied in subsequent experiments.

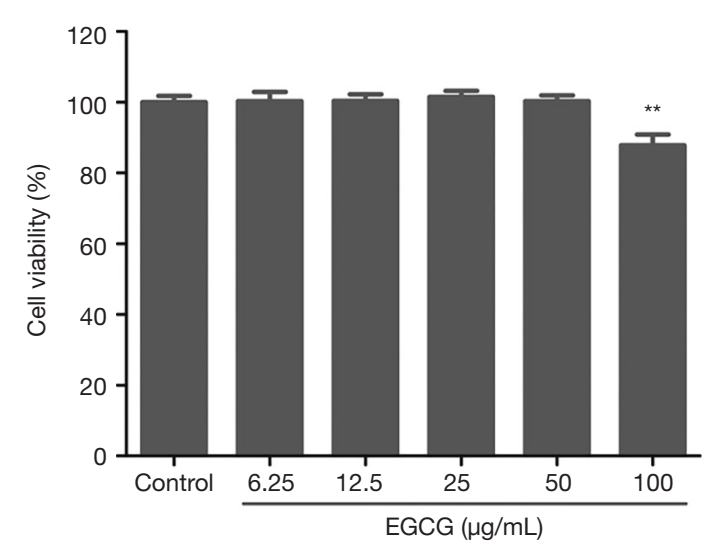

Figure 1 The BEAS-2B cells viability after treatment with EGCG for 48 h. *, $\mathrm{P}<0.05$; **, $\mathrm{P}<0.01$ vs. control group. EGCG, (-)-epigallocatechin-3-gallate.

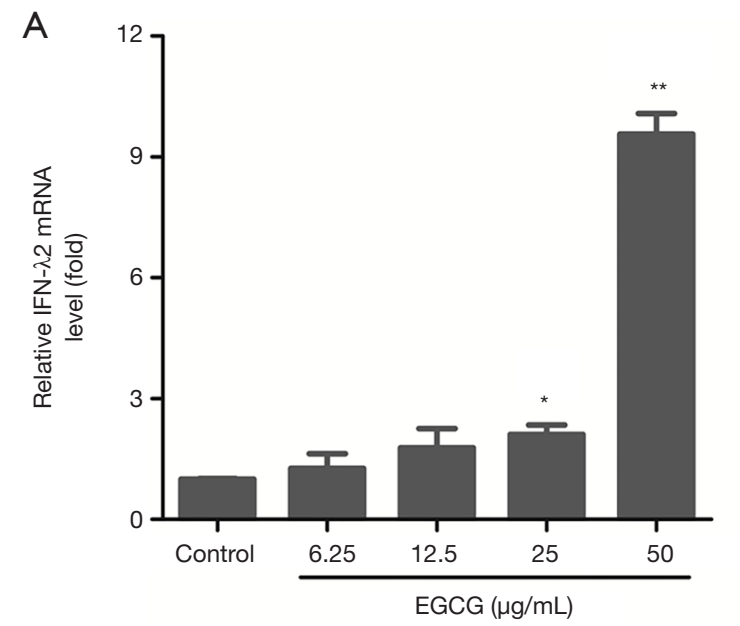

\section{EGCG induces IFN- 22 expression in BEAS-2B cells}

After detecting a concentration range in which EGCG is not cytotoxic to BEAS-2B cells, we next sought to determine the time and dose dependency that EGCG induces IFN- $\lambda 2$ expression in BEAS-2B cells. Cells were treated with EGCG. The expression level of the IFN- $\lambda 2$ mRNA was analyzed by qRT-PCR, and expression level of the IFN- $\lambda 2$ protein was analyzed by ELISA. We found that both IFN- $\lambda 2$ mRNA and protein levels were significantly up-regulated in BEAS-2B cells in a dose- and time-dependent manner after being treated with EGCG (Figures 2,3).

\section{EGCG induces IFN- $\lambda 2$ expression in BEAS-2B cells by $p 38$ MAPK signaling pathway}

As the stimulation with $50 \mu \mathrm{g} / \mathrm{mL}$ of EGCG showed the strongest response in inducing the expression of IFN- $\lambda 2$, we next selected $50 \mu \mathrm{g} / \mathrm{mL}$ EGCG to determine the signal pathways in which EGCG induces IFN- $\lambda 2$ expression in BEAS-2B cells. BEAS-2B cells were treated with $50 \mu \mathrm{g} / \mathrm{mL}$ EGCG for $0,2,4,12,24$, and $48 \mathrm{~h}$, and we then determined the effect of EGCG on the activation of ERK, p38 MAPK, and JNK in BEAS-2B cells by western blot. As shown in Figure 4, the total ERK, p38 MAPK, and JNK levels had no effect after $50 \mu \mathrm{g} / \mathrm{mL}$ of EGCG treatment, but the phosphorylated (activation) ERK, p38 MAPK, and

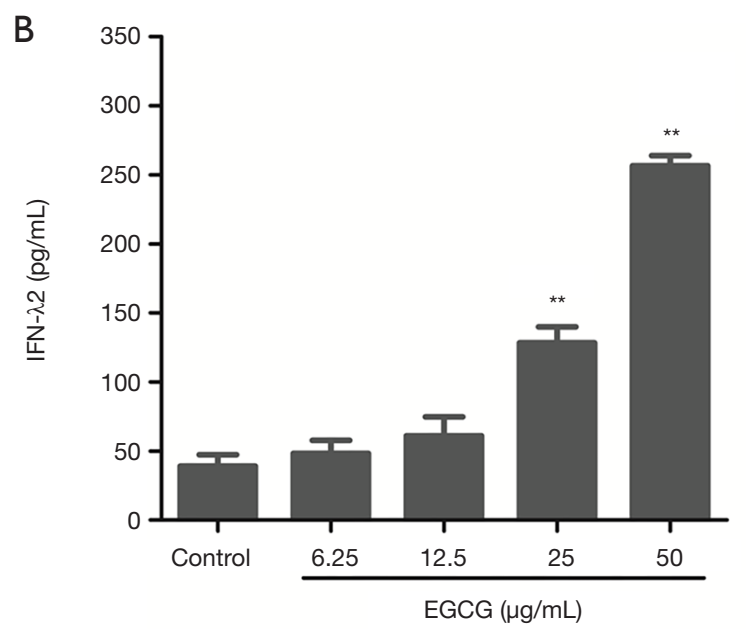

Figure 2 The IFN- $\lambda 2$ expression in BEAS-2B cells after treatment with EGCG for 12 h. (A) IFN- $\lambda 2$ mRNA expression; (B) IFN- $\lambda 2$ protein expression. * $\mathrm{P}<0.05$; **, $\mathrm{P}<0.01$ vs. control group. IFN, interferon; EGCG, (-)-epigallocatechin-3-gallate. 

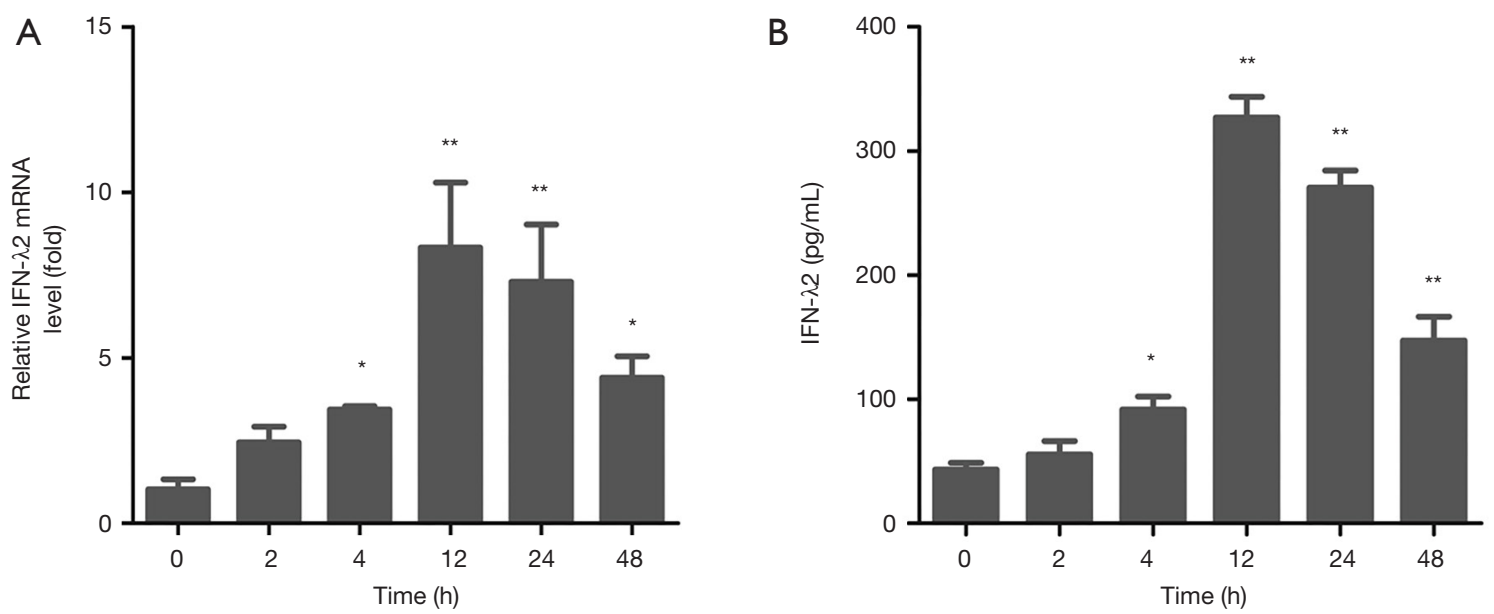

Figure 3 The IFN- $\lambda 2$ expression in BEAS-2B cells after treatment with EGCG $(50 \mu \mathrm{g} / \mathrm{mL})$ for $0,2,4,12,24$, and 48 h. (A) IFN- $\lambda 2 \mathrm{mRNA}$ expression; (B) IFN- $\lambda 2$ protein expression. *, $\mathrm{P}<0.05$; **, $\mathrm{P}<0.01$ vs. 0 h group. IFN, interferon; EGCG, (-)-epigallocatechin-3-gallate.

A

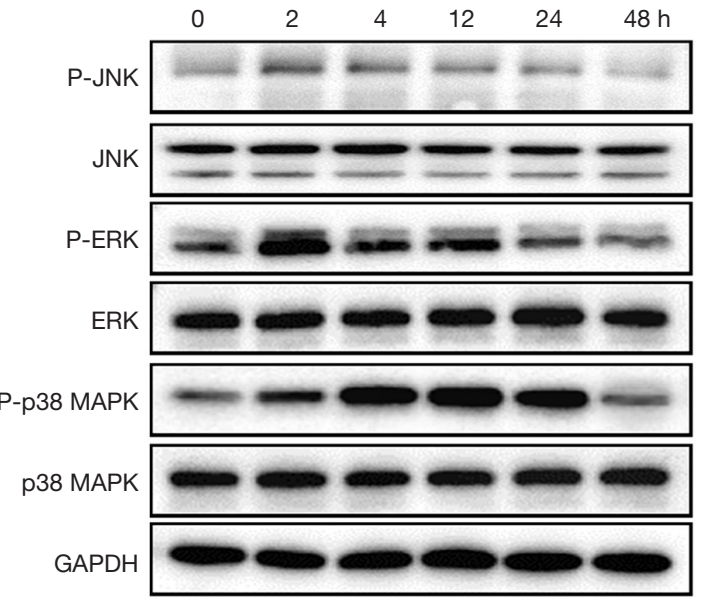

C

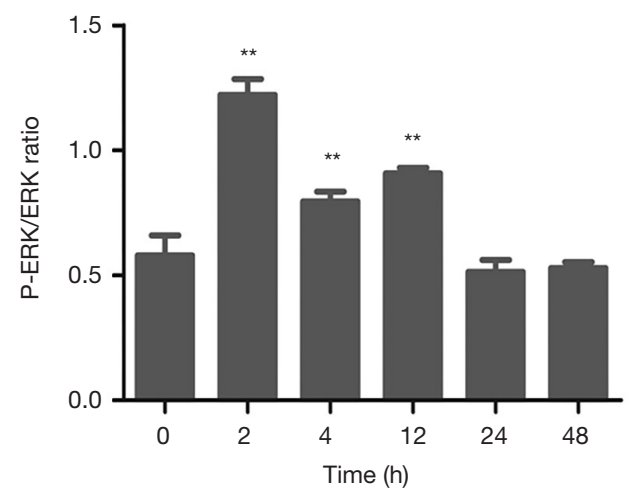

B

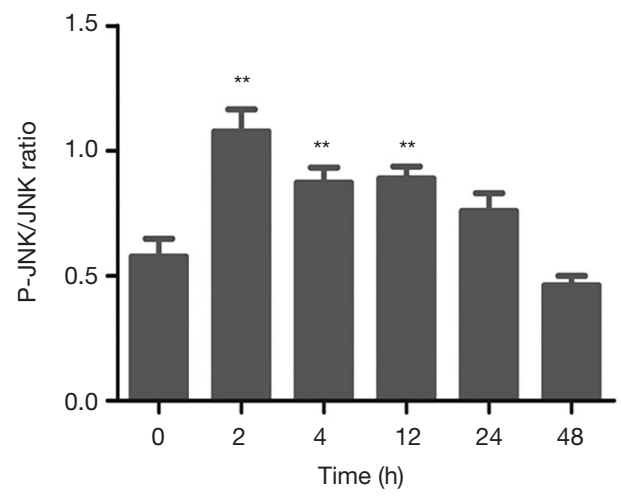

D

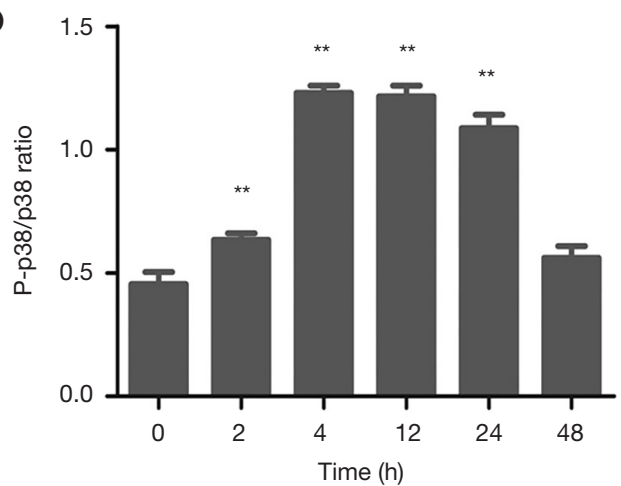

Figure 4 The P-p38 MAPK, P-ERK, and P-JNK expression in BEAS-2B cells after treatment with EGCG $(50 \mu \mathrm{g} / \mathrm{mL})$ for 0, 2, 4, 12, 24 , and $48 \mathrm{~h}$. (A) The western blotting map of P-p38 MAPK, P-ERK, and P-JNK; (B) P-JNK protein expression; (C) P-p38 MAPK protein expression; (D) P-p38 MAPK protein expression. *, $\mathrm{P}<0.05$; ** $\mathrm{P}<0.01$ vs. 0 h group. P-p38 MAPK, phospho-p38 mitogen-activated protein kinase; P-ERK, phosphoextracellular signal-regulated kinase; P-JNK, phospho-c-Jun N-terminal kinase; EGCG, (-)-epigallocatechin-3-gallate. 
A

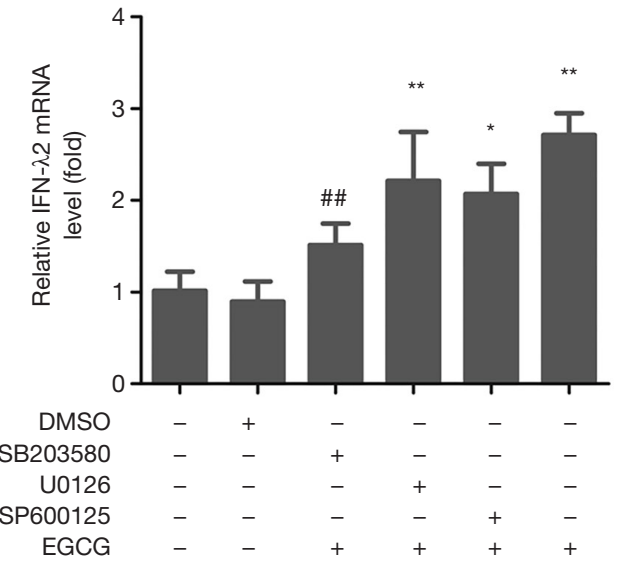

B

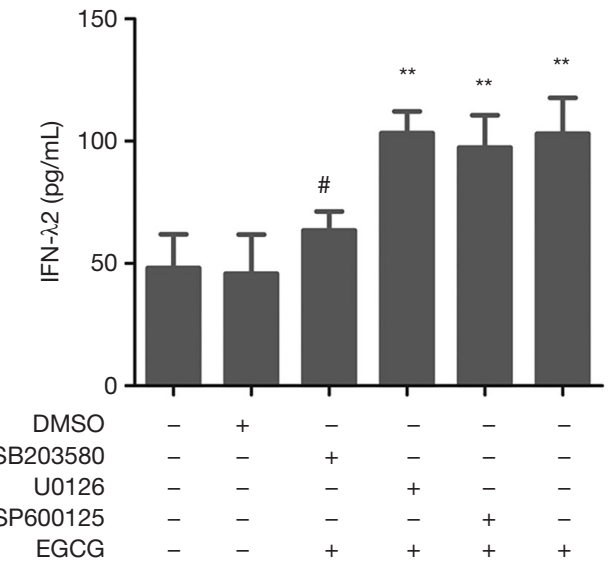

Figure 5 The IFN- $\lambda 2$ expression in BEAS-2B cells. (A) IFN- $\lambda 2$ mRNA expression; (B) IFN- $\lambda 2$ protein expression. *, $\mathrm{P}<0.05$; **, $\mathrm{P}<0.01 v s$. control group; \#, $\mathrm{P}<0.05$; \#\#, $\mathrm{P}<0.01$ vs. EGCG group. EGCG, (-)-epigallocatechin-3-gallate; IFN- $\lambda 2$, interferon-lambda2.

JNK levels were significantly up-regulated. The activation time of ERK and JNK occurred at 2 to $12 \mathrm{~h}$, and that of $\mathrm{p} 38$ MAPK occurred at 2 to $24 \mathrm{~h}$, following the treatment with EGCG. The result confirmed that EGCG can activate p38 MAPK, ERK, and JNK signaling pathways in BEAS-2B cells (Figure 4). Next, we tested the functions of p38 MAPK, ERK, and JNK signaling pathways in EGCG-induced IFN- $\lambda 2 \mathrm{mRNA}$ and protein expression. The BEAS-2B cells were pretreated with $20 \mu \mathrm{M}$ SB203580, $20 \mu \mathrm{M}$ U0126, or $10 \mu \mathrm{M}$ SP600125 for $1 \mathrm{~h}$, after which time the $50 \mu \mathrm{g} / \mathrm{mL}$ of EGCG was mixed with the cells for $4 \mathrm{~h}$. We found that pretreatment with SB203580 significantly inhibited EGCGinduced IFN- $\lambda 2$ mRNA and protein expression, whereas pretreatment with U0126 and SP600125 did not show inhibition (Figure 5).

\section{EGCG inbibits IAV H1N1 replication in BEAS-2B cells by inducing IFN- $\lambda 2$ expression}

The results above confirmed that EGCG can induce the expression of IFN- $\lambda 2$ in BEAS-2B cells. We next tested whether EGCG's anti-IAV (H1N1) effect was related to the induced IFN- $\lambda 2$ expression. The BEAS-2B cells were pretreated with $50 \mu \mathrm{g} / \mathrm{mL}$ EGCG, $50 \mu \mathrm{g} / \mathrm{mL}$ EGCG and $0.5 \mu \mathrm{g} / \mathrm{mL}$ IFN- $\lambda 2$ neutralizing antibody, or $50 \mu \mathrm{g} / \mathrm{mL}$ EGCG and $0.5 \mu \mathrm{g} / \mathrm{mL}$ control antibody for $12 \mathrm{~h}$, and then the cells were infected with $100 \times \mathrm{TCID}_{50} \mathrm{H} 1 \mathrm{~N} 1$ for $1 \mathrm{~h}$. The expression levels of the H1N1 NP gene and protein was analyzed by qRT-PCR and western blot, respectively. The results showed that EGCG significantly downregulated the H1N1 NP gene and protein expression, while IFN- $\lambda 2$ neutralizing antibody attenuated the effect of EGCG's down-regulation of the H1N1 NP gene and protein expression (Figure 6).

\section{Discussion}

Green tea has been regarded as a health product for more than 2000 years and is a major source of polyphenol antioxidants $(20,21)$. Green tea catechins, especially the main catechin found in green tea, epigallocate-chin-3gallate (EGCG) have demonstrated anti-viral and antiinflammatory properties (22). In the previous study, EGCG was found to inhibit influenza viral replication by damaging the physical properties of the viral envelope and partial inhibition of the NA surface glycoprotein (19). However, the immune regulation mechanisms of EGCG' anti-IAV effects remain unclear. To our knowledge, this is the first study to demonstrate that EGCG up-regulates IFN- $\lambda 2$ expression through p38 MAPK signaling pathway and inhibits IAV H1N1 replication in BEAS-2B cells.

The IFN- $\lambda$ family, also known as type III IFN, includes the IFN- $\lambda 1$, IFN- $\lambda 2$, and IFN- $\lambda 3$ members $(22,23)$. In mice, only IFN- $\lambda 2$ and IFN- $\lambda 3$ are expressed (24). IFN- $\lambda$ plays an important role in combating pathogen infection, has been shown to have protective effects against IAV infection, and to contribute to local anti-IAV innate immunity $(25,26)$. More excitingly, the effect of IFN $-\lambda$ against the IAV is better than that of type I IFN (27). However, the cost of directly using IFN- $\lambda$ to treat influenza virus is high, and plant extracts have the advantage of low toxicity, easy availability, and being economical. In the previous 
(
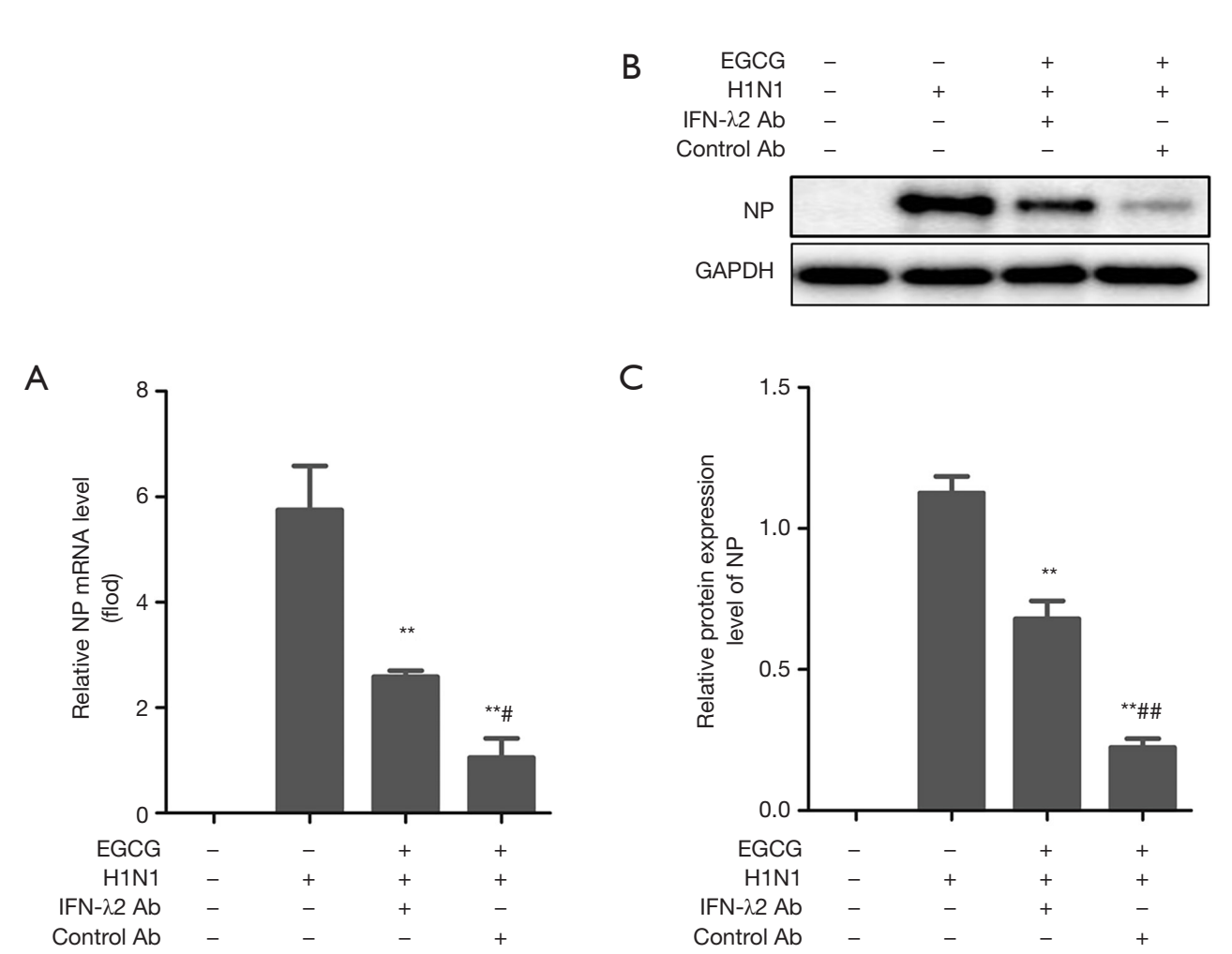

Figure 6 The NP expression in BEAS-2B cells. (A) NP mRNA expression; (B) the western blotting map of NP; (C) NP protein expression. *, $\mathrm{P}<0.05$; ** $\mathrm{P}<0.01$ vs. $\mathrm{H} 1 \mathrm{Nl}$ group; $\#, \mathrm{P}<0.05$; \#\#, $\mathrm{P}<0.01$ vs. IFN- $\lambda 2$ Ab group. $\mathrm{NP}$, nucleoprotein; IFN, interferon.

study, the crude phenylethanoid glycosides isolated from Ligustrum purpurascens via inducing endogenous IFN- $\gamma$ had an anti-viral effect against the IAV (28). In other studies, EGCG inhibited HCV replication by enhancing virus dsRNA or poly I:C-induced IFN- $\lambda 1$ production $(17,18)$. Consequently, these studies provided us with new ideas. In the present study, we used EGCG to treat BEAS-2Bs, with the results showing that EGCG significantly up-regulated IFN- $\lambda 2$ mRNA and protein levels in BEAS-2B cells. Therefore, we confirmed that EGCG can induce IFN- $\lambda 2$ production in BEAS-2B cells.

In addition, we also explored the mechanisms of EGCG-induced IFN $\lambda 2$ expression in BEAS-2B cells. The MAPKs in mammals include c-Jun NH2-terminal kinase (JNK), p38 mitogen-activated protein kinase (p38 MAPK), and extracellular signal-regulated kinase (ERK). These enzymes are serine-threonine protein kinases that regulate various cellular activities including proliferation, differentiation, apoptosis or survival, inflammation, and innate immunity (29). EGCG treatment was able to induce NB4 cell apoptosis by increasing phosphorylated (p)-p38 $\alpha$ MAPK expression (30). In addition, other studies have found that EGCG also regulates ERK and JNK signaling pathways $(31,32)$. In the present study, we found that EGCG could significantly increase P-p38 MAPK, P-ERK, and P-JNK levels, and activate p38 MAPK, ERK, and JNK signaling pathways. Furthermore, p38 MAPK, ERK, or JNK inhibitor was used to inhibit signaling pathways: p38 MAPK inhibitor significantly decreased the EGCGinduced expression level of IFN- $\lambda 2$ in BEAS-2B cells, while the ERK and JNK inhibitors had no effects. Our result is similar to the other study which also has demonstrated that IFN- $\lambda 1$ was regulated by p38 MAPK $\alpha$ (33). Accordingly, our study has demonstrated that EGCG can induce the expression of IFN- $\lambda 2$ through the p38 MAPK signaling pathway in BEAS-2B cells.

Besides, EGCG pretreatment significantly inhibited the expression levels of IAV H1N1 NP gene and protein in BEAS-2B cells. However, IFN- $\lambda 2$ neutralizing antibody attenuated the effect of EGCG inhibiting the expression levels of IAV H1N1 NP gene and protein. Combining our new findings with other research results, EGCG not only inhibits influenza viral replication by damage to the physical properties of the viral envelope and partial 
inhibition of the NA surface glycoprotein (19) but also anti-IAV by inducing IFN- $\lambda 2$ expression. A recent study demonstrated that EGCG could treat acute lung injury induced by swine influenza virus (H9N2) through 67LR/ Tollip-downregulated TLR4 protein levels, decreased MPO activity and inflammatory cytokine levels (34). These findings suggest that EGCG can fight influenza viruses through a variety of mechanisms.

\section{Conclusions}

Our study demonstrated that EGCG can inhibit IAV H1N1 replication by inducing IFN- $\lambda 2$ expression through $\mathrm{p} 38$ MAPK signaling pathway in BEAS-2B cells. Its effective regulation of IAV H1N1 replication implies that EGCG is a potential alternative treatment for the IAV.

\section{Acknowledgments}

Funding: None.

\section{Footnote}

Conflicts of Interest: The authors have no conflicts of interest to declare.

Ethical Statement: The authors are accountable for all aspects of the work in ensuring that questions related to the accuracy or integrity of any part of the work are appropriately investigated and resolved.

Open Access Statement: This is an Open Access article distributed in accordance with the Creative Commons Attribution-NonCommercial-NoDerivs 4.0 International License (CC BY-NC-ND 4.0), which permits the noncommercial replication and distribution of the article with the strict proviso that no changes or edits are made and the original work is properly cited (including links to both the formal publication through the relevant DOI and the license). See: https://creativecommons.org/licenses/by-nc-nd/4.0/.

\section{References}

1. Hutchinson EC. Influenza Virus. Trends Microbiol 2018;26:809-10.

2. Jiang WM, Wang SC, Peng C, et al. Identification of a potential novel type of influenza virus in Bovine in China. Virus Genes 2014;49:493-6.
3. Yandrapalli S, Aronow WS, Frishman WH. Readmissions in adult patients following hospitalization for influenza: a nationwide cohort study. Ann Transl Med 2018;6:318.

4. Nagesh PT, Mazhar H, Galvin HD, et al. Histone Deacetylase 2 Is a Component of Influenza A VirusInduced Host Antiviral Response. Front Microbiol 2017;8:1315-26.

5. Dong G, Peng C, Luo J, et al. Adamantane-Resistant Influenza A Viruses in the World (1902-2013): Frequency and Distribution of M2 Gene Mutations. Plos One 2015;10:e0119115.

6. McKimm-Breschkin JL. Influenza neuraminidase inhibitors: antiviral action and mechanisms of resistance. Influenza Other Respir Viruses 2013;7:25-36.

7. Hussain M, Galvin HD, Haw TY, et al. Drug resistance in influenza A virus: the epidemiology and management. Infect Drug Resist 2017;10:121-34.

8. Baturcam E, Vollmer S, Schlüter H, et al. MEK inhibition drives anti-viral defence in RV but not RSV challenged human airway epithelial cells through AKT/p70S6K/4EBP1 signalling. Cell Commun Signal 2019;17:78-96.

9. Commins SP, Borish L, Steinke JW. Immunologic messenger molecules: Cytokines, interferons and chemokines. J Allergy Clin Immunol 2010;125:S53-72.

10. Nogales A, Martinez-Sobrido L, Topham D, et al. Modulation of Innate Immune Responses by the Influenza A NS1 and PA-X Proteins. Viruses 2018. doi: 10.3390/ v10120708.

11. Vázquez Cisneros LC, López-Uriarte P, López-Espinoza A, et al. Effects of green tea and its epigallocatechin (EGCG) content on body weight and fat massin humans: a systematic review. Nutr Hosp 2017;34:731-7.

12. Castellano LM, Hammond RM, Holmes VM, et al. Epigallocatechin-3-gallate rapidly remodels PAP85120, SEM1(45-107), and SEM2(49-107) seminal amyloid fibrils. Biol Open 2015;4:1206-12.

13. Shivani NP, Sandra DA, Lee HL et al. Inhibition of Herpes Simplex Virus-1 by the Modified Green Tea Polyphenol EGCG-Stearate. ABB 2018;9:679-90.

14. Lai YH, Sun C2, Huang HC, et al. Epigallocatechin gallate inhibits hepatitis B virus infection in human liver chimeric mice. BMC Complement Altern Med 2018; $18: 248$.

15. Calland N, Albecka A, Belouzard S, et al. (-)-Epigallocatechin-3-gallate is a new inhibitor of hepatitis C virus entry. Hepatology 2012;55:720-9.

16. Sharma N, Murali A, Singh SK, et al. Epigallocatechin gallate, an active green tea compound inhibits the Zika 
virus entry into host cells via binding the envelope protein. Int J Biol Macromol 2017;104:1046-54.

17. Wang Y, Li J, Wang X, et al. (-)-Epigallocatechin-3-Gallate Enhances Hepatitis C Virus Double-Stranded RNA Intermediates-Triggered Innate Immune Responses in Hepatocytes. Sci Rep 2016;6:21595-606.

18. Wang YZ, Li JL, Wang X, et al. (-)-Epigallocatechin3 -gallate enhances poly I:C-induced interferon- $\lambda 1$ production and inhibits hepatitis $\mathrm{C}$ virus replication in hepatocytes. World J Gastroenterol 2017;23:5895-903.

19. Ide K, Kawasaki Y, Kawakami K, et al. Anti-influenza Virus Effects of Catechins: A Molecular and Clinical Review. Curr Med Chem 2016;23:4773-83.

20. Chacko SM Thambi PT, Kuttan R, et al. Beneficial effects of green tea: A literature review. Chin Med 2010;5:13-21.

21. Hu J, Gu J, Xu F, et al. Epigallocatechin-3-gallate Attenuates Myocardial Reperfusion Injury in Rats Through Activation of PI3K/Akt Signaling Pathway. Sichuan Da Xue Xue Bao Yi Xue Ban 2016;47:305-9.

22. Kotenko SV, Gallagher G, Baurin VV, et al. IFN-lambdas mediate antiviral protection through a distinct class II cytokine receptor complex. Nat Immunol 2003;4:69-77.

23. Sheppard P, Kindsvogel W, Xu W, et al. IL-28, IL-29 and their class II cytokine receptor IL-28R. Nat Immunol 2003;4:63-8.

24. Lasfar A, Lewisantes A, Smirnov SV, et al. Characterization of the mouse IFN-lambda ligand-receptor system: IFNlambdas exhibit antitumor activity against B16 melanoma. Cancer Res 2006;66:4468-77.

25. Klinkhammer J, Schnepf D, Ye L, et al. IFN- $\lambda$ prevents influenza virus spread from the upper airways to the lungs and limits virus transmission. Elife 2018: doi: 10.7554/ eLife.33354.

26. Kim S, Kim MJ, Kim CH, et al. The Superiority of IFNlambda as a Therapeutic Candidate to Control Acute

Cite this article as: Zhu J, Ou L, Zhou Y, Yang Z, Bie M. (-)-Epigallocatechin-3-gallate induces interferon- $\lambda 2$ expression to anti-influenza A virus in human bronchial epithelial cells (BEAS-2B) through p38 MAPK signaling pathway. J Thorac Dis 2020;12(3):989-997. doi: 10.21037/jtd.2020.03.20
Influenza Viral Lung Infection. Am J Respir Cell Mol Biol 2017;56:202-12.

27. Davidson S, Mccabe TM, Crotta S, et al. IFN- $\lambda$ is a potent anti-influenza therapeutic without the inflammatory side effects of IFN- $\alpha$ treatment. EMBO Mol Med 2016;8:1099-112.

28. Hu XP, Shao MM, Song X, et al. Anti-influenza virus effects of crude phenylethanoid glycosides isolated from ligustrum purpurascens via inducing endogenous interferon- $\gamma$. J Ethnopharmacol 2016;179:128-36.

29. Kim EK, Choi EJ. Compromised MAPK signaling in human diseases: an update. Arch Toxicol 2015;89:867-82.

30. Gan L, Zhong L, Shan Z, et al. Epigallocatechin-3gallate induces apoptosis in acute promyelocytic leukemia cells via a SHP-1-p38 $\alpha$ MAPK-Bax cascade. Oncol Lett 2017;14:6314-20.

31. Satonaka H, Ishida K, Takai M, et al. (-)-Epigallocatechin3-gallate Down-regulates Doxorubicin-induced Overexpression of P-glycoprotein Through the Coordinate Inhibition of PI3K/Akt and MEK/ERK Signaling Pathways. Anticancer Res 2017;37:6071-7.

32. Xiao X, Jiang K, Xu Y, et al. (-)-Epigallocatechin-3-gallate induces cell apoptosis in chronic myeloid leukaemia by regulating Bcr/Abl-mediated p38-MAPK/JNK and JAK2/ STAT3/AKT signalling pathways. Clin Exp Pharmacol Physiol 2019;46:126-36.

33. Jiang M, Osterlund P, Fagerlund R, et al. MAP kinase p38 regulates type III interferon (IFN- $\lambda$ ) gene expression in human monocyte-derived dendritic cells in response to RNA stimulation. J Leukoc Biol 2015;97:307-20.

34. Xu MJ, Liu BJ, Wang CL, et al. Epigallocatechin-3-gallate inhibits TLR4 signaling through the $67-\mathrm{kDa}$ laminin receptor and effectively alleviates acute lung injury induced by H9N2 swine influenza virus. Int Immunopharmacol 2017;52:24-33. 